\title{
Large Ejaculatory Duct Cyst in 48years old man: Open Surgery and Fertility Issues
}

\author{
Syed Sultan Mahmud ${ }^{1}$, Md. Saiful Islam ${ }^{2}$, Md. Mostafizur Rahaman ${ }^{3}$, Manas Majumder ${ }^{4}$, Mohammad \\ Shafiqur Rahman ${ }^{5}$, Md. Saydul Islam ${ }^{6}$, Mohammad Hossain ${ }^{7}$
}

Received: 05 - 06 - 2020

Accepted: $010-08-2020$

Conflicts of interest: None

Keywords: Ejaculatory, Duct, Cyst.

\begin{abstract}
Pelvic cyst originated from Mullerian or Wolffian duct are reported frequently but ejaculatory duct cysts are very rare. Here we present a rare case of large ejaculatory duct cyst in a diabetic man. In Bangladesh no such case has yet reported. This will be the first case reported in our country. This man was 48 years old presented with post ejaculatory pain, haematospermia, low ejaculatory volume and infertility. On trans rectal ultrasonogram (TRUS) there was $70 \mathrm{ml}$ volume retro prostatic midline cyst was found. MRI pelvis confirms the diagnosis.
\end{abstract}

There are different modalities of treatment ranging from observation to open surgery. We chose to open excision with good post operative outcome.

Ejaculatory duct cyst is a rare entity. Malignant transformation also reported in ejaculatory duct cyst. Trans rectal ultra sonogram (TRUS) and MRI of pelvic organs are diagnostic. Treatment depends on size of cyst and fertility. Total excision result in complete recovery from symptoms and disease. Transurethral surgery may conserve natural fertility but persistence in complications. Fertility issue in our case depends on intra-cytoplasmic sperm injection which outcome will be seen in future follow up.

\section{Introduction}

Cystic anomalies of the Wolffian and Mullerian duct systems presenting clinically in men are uncommon and the differential diagnosis can be a dilemma to the urologist. ${ }^{1}$ Urogenital sinus cysts are deep midline pelvic cysts or retrovesical, characterized by communicating with both Wolffian systems (vas deferens and seminal vesicles and ejaculatory ducts). ${ }^{2}$

There are two ejaculatory duct opened separately in verumontanum. The initial segments of bilateral ejaculatory ducts are very slender and their locations are adjacent. Thus, local tiny lesions can cause bilateral ejaculatory duct obstruction. ${ }^{3}$
Ejaculatory duct obstruction may be caused by congenital cyst of the prostate, utricle, seminal vesicle, ejaculatory duct, mullerian duct or secondary to tuberculosis, chronic prostatitis, surgery (transurethral, pull-through anoplasty), gunshot wound, urethral foreign body. ${ }^{2}$

A small volume ejaculate with low $\mathrm{pH}$ and absent fructose but with palpable Vasa is virtually pathognomic. ${ }^{5}$ Although semen fructose can provide presumptive evidence of ejaculatory duct obstruction or seminal vesicle atresia, this test yield little information regarding individual patient anatomy.

1. Post Graduate Student (MS Urology), Department of Urology, BSMMU, Dhaka

2. Assistant Professor, Department of Urology, BSMMU, Dhaka

3. Medical Officer, Department of Urology, BSMMU, Dhaka

4. Post Graduate student (MS Urology), Department of Urology, BSMMU, Dhaka

5. Associate Professor, Department of Urology, BSMMU, Dhaka

6. Associate Professor, Department of Urology, BSMMU, Dhaka

7. Associate Professor, Department of Urology, BSMMU, Dhaka

Correspondence: Dr. Syed Sultan Mahmud, Post Graduate Student (MS Urology), Department of Urology, BSMMU, Dhaka-1000, E-mail: syedsultanamahmud29@gamil.com 
Ultrasonography established the diagnosis of ejaculatory duct obstruction and also demonstrated the exact distal extent of the dilated system into the prostate. ${ }^{7}$

Vasography is a "gold standard" for the diagnosis of the ejaculatory duct obstruction. But it is an invasive method. ]MRI can accurately display the ejaculatory duct and lesions. ${ }^{3}$ The proper surgical approach to genital duct cysts depends on the location and size of the cyst, as well as the age of the patient. Preservation of sexual potency and fertility is of the utmost importance. ${ }^{8}$

\section{Case report}

This 48 years old man presented to us with primary infertility with low ejaculatory volume for 17 years and haematospermia with post ejaculatory pain for 5 years. Patient was married for 18 years having three wives but no issue. He is diabetic and taking insulin for 11 years.

Post ejaculatory pain evident as lower abdominal and urethral pain mild in nature and resolve spontaneously. Haematospermia present in 30 percent times of his seminal ejaculation. He has normal penile erection and sexual relationship. Bladder and bowel habit was quite normal.

Physical finding was unremarkable except a boggy feeling cystic swelling felt above the prostate which can't be outlined digitally.

Investigations revealed- semen analysis: alkaline, low volume, absence of sperm; Serum testosterone, luteinizing hormone, follicle-stimulating hormone and prolactin levels were normal; FNA both testes: normal spermatogenesis; transrectal ultrasonogram: big cyst in posterior aspect of prostate appear to be ejaculatory

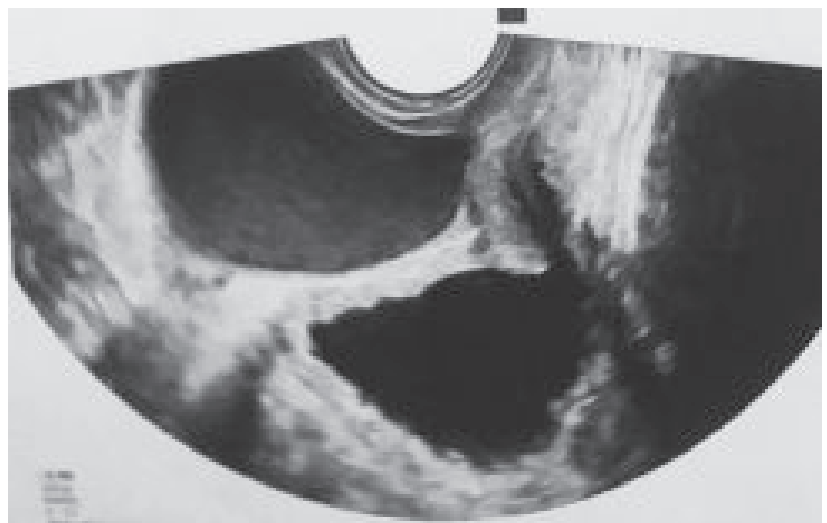

Fig.-1: Transrectal ultrasonogram view of cyst.

duct origin measuring $63 \times 53 \times 43 \mathrm{~mm}(76 \mathrm{ml})$ (Fig:1); MRI: large oval cyst $51 \times 52 \times 72 \mathrm{~mm}$ in right lateral aspect of prostate, posterior to bladder and anterior to rectum with mild marginal enhancement (Fig:2).

Operation done with detailed preoperative counseling. On urethrocystoscopy verumontanum and prostatic utricle seen. Ejaculatory duct openings were not seen. There was normal looking bladder and ureteric orifices. Bladder was emptied by urethral catheter after induction of anesthesia. Abdomen opened by lower midline incision. Large cyst identified in between bladder and rectum. Cyst fluid aspirated and examined under microscope where dead spermatozoa found (Fig:3). Space created around the cyst by sharp and blant dissection. Two seminal vesicle and vas found normal and drained into the dome of the cyst (Fig:4). Total cyst was excised with bilateral vasoligation (Fig:5).

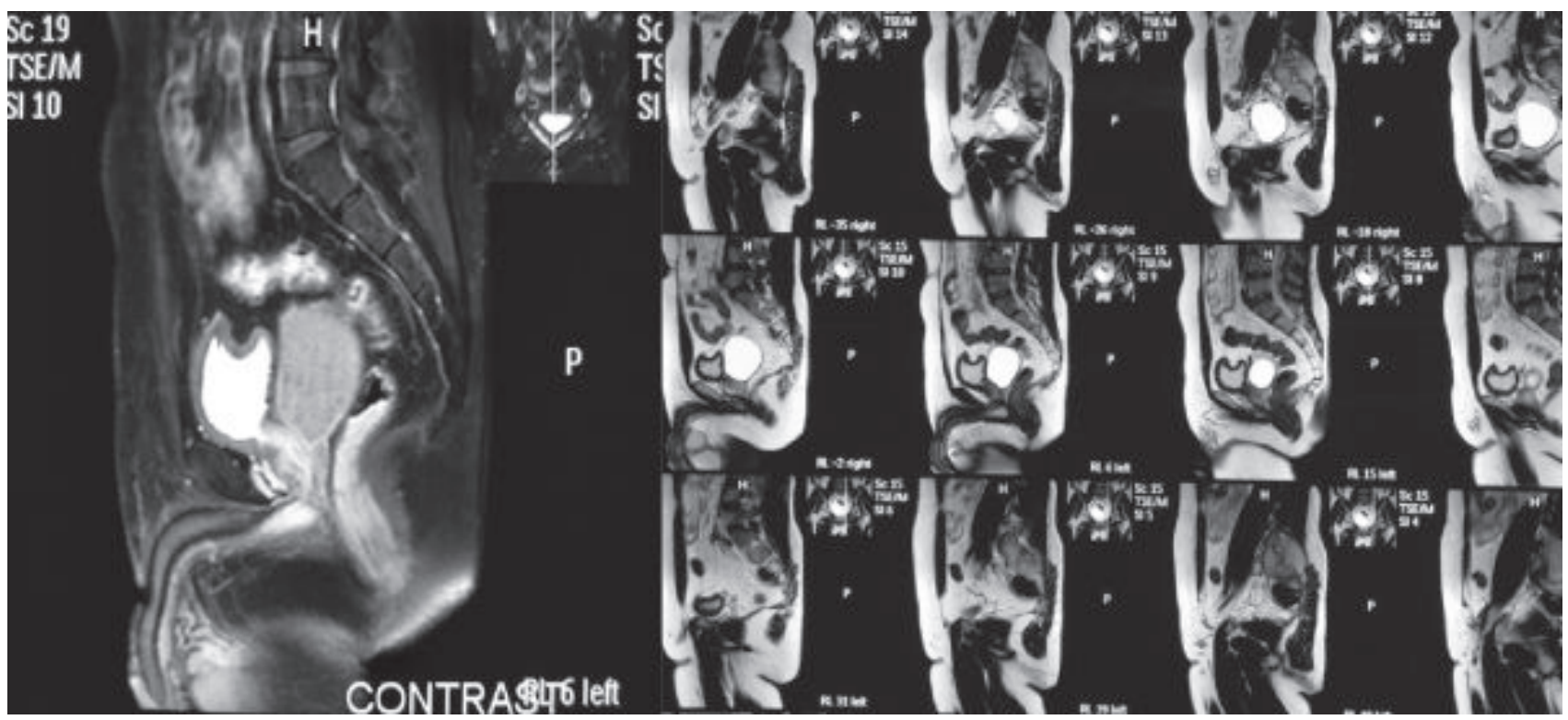

Fig.-2: MRI pelvis T2 weighted with I/V Gadolinium contrast. 


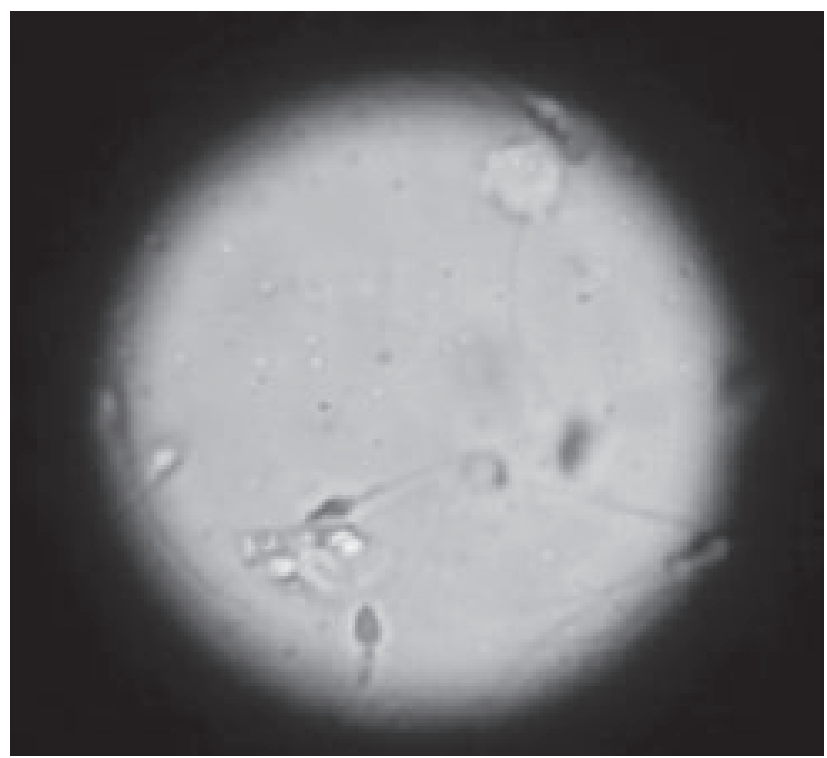

Fig.-3: Intraoperative microscopy showing dead spermatozoa.

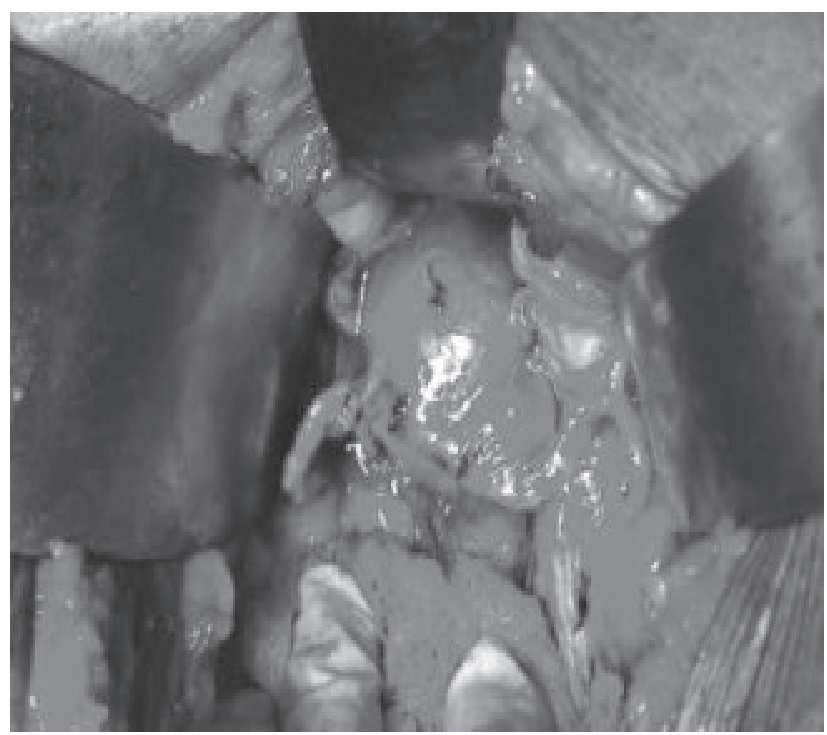

Fig.-4: Intraoperative view of ejaculatory cyst. Two vas opened into the cyst.

Post operative recovery was uneventful. Patient discharged on $6^{\text {th }}$ post operative day. He is asymptomatic at present and didn't presented alterations in the sexual sphere.

\section{Discussion}

Embryologically vas-defferens, seminal vesicles and ejaculatory duct derived from mesonephric duct during 8th weeks of embryo. Two mullerian structure persists in male- appendix of testes and prostatic utricle. Two ejaculatory duct drained separately in

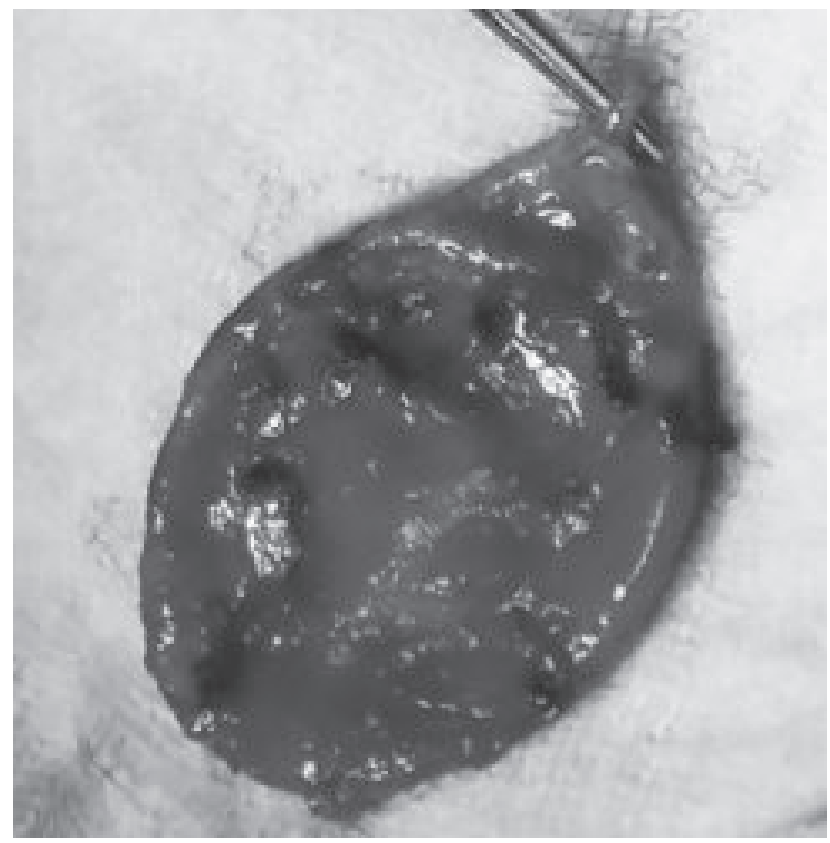

Fig.-5: Total excised cyst with ejaculatory duct.

verumontanum which openings seen on urethrocystoscopy. Routine vasography revealed ejaculatory duct obstruction in 5\% of azoospermic men. ${ }^{9}$

In this case we found one ejaculatory duct system. Cystic structure in this region with infertility issue was classified by Mayersak. ${ }^{1}$

The possibility of a cystic lesion of the internal male genitalia is usually entertained after an atypical mass is palpable on rectal examination. ${ }^{10}$

It is impossible to determine whether the etiology of the ejaculatory duct cyst in our case was congenital or iatrogenic.

Patient can present with watery low semen volume, post ejaculatory pain, haematospermia and infertility. Patient having normal secondary sexual characteristics. Diagnosis made by semen analysis low volume, acidic semen, absence of fructose and sperm. Transrectal ultrasonogram can identify cystic structure in retrovesical position. For better delineation MRI of pelvis necessary. Patient having normal blood hormonal parameters and spermatogenesis on testicular biopsy. There are various treatment modalities available: Aspiration of cyst: chance of recurrence and pelvic abscess. ${ }^{8}$

Dilatation of the ejaculatory duct by F9 seminal vesicoscopy is a novel procedure.[11] TURED: chance 


\section{Classification of cystic masses occurring behind bladder Cystic mass lesion behind the bladder}

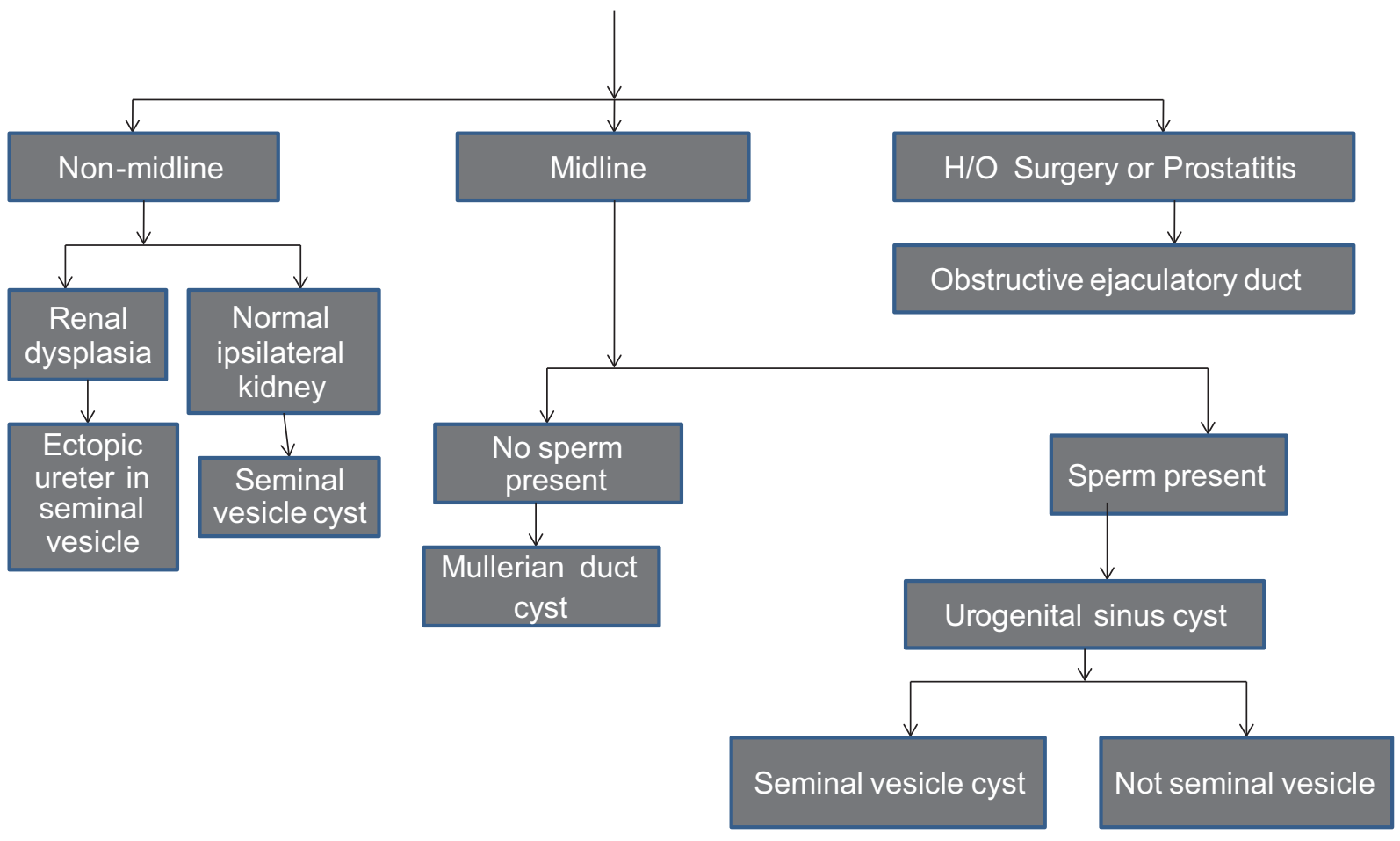

Fig.-6: Classification of cystic masses by Mayersak (1989) ${ }^{[1]}$.

of recurrent epididymo-orchitis, urinary incontinence from external sphincter injury, retrograde ejaculation from bladder neck incision, rectal injury, urinary dribbling from urinary intravasation into the cyst from prostatic urethra after de-roofing, recurrent stenosis and cyst formation. ${ }^{9,11}$ Complication rate is $20 \%$. Goluboff and his associates showed dribbling of urine after TURED. ${ }^{12}$ Obstructions secondary to genital infections seem not to be amenable to this treatment, with none of the patient achieving normal sperm counts or pregnancy. ${ }^{13}$

Total excision of cyst: laparoscopy or open excision chance of erectile dysfunction, permanent infertility. We preferred open extravesical excision. Which is relative easy and complication free. But fertility by natural route may not possible. Fertility possible by ICSI. Metaplastic change occurs in ejaculatory duct cyst. Toshihiro and his associate found metaplastic change in ejaculatory duct cyst and was treated open excision with bilateral vasoligation. ${ }^{14}$

\section{Conclusion}

Our patient having large $75 \mathrm{ml}$ ejaculatory duct cyst and interestingly two seminal vesicles opened this single cyst. We chose to do open excision as because large cyst and avoiding complications which commonly occur by transurethral resection. Fertility through natural route not possible in this case. Patient was advised about ICSI.

\section{References}

1. Mayersak J. S. Urogenital sinus-ejaculatory duct cyst: A case report with a proposed clinical classification and review of the literature. Journal of Urology 1989; 142:1330-1332.

2. Felipe SB, Bernardo HI, Francisco AC, Elisabeth CG, Andre's CM, Ana YG, et al. Urogenital Sinus Cyst in a 21-Year-Old Man. Arch Sex Behav 2012; 41:1065-1068.

3. Yan G, Guihua L, Dong Y, Xiangzhou S, Huanjun W, Chunhua D, et al. Role of MRI in assessment of ejaculatory duct obstruction . Journal of X-Ray Science and Technology 2013; 21: 141-146.

4. Ira D, Sharlip M. Obstructive azoospermia or oligozoospermia due to Mullerian duct cyst. Fertility and Sterility (1984)41: 298-303. 
5. John P. and Henry F Ejaculatory duct obstruction in subfertile males: analysis of 87 patients. Fertility and sterility(1991)56: 725-730.

6. Meacham B, Hellerstein K and Lipshultz I. Evaluation and treatment of ejaculatory duct obstruction in the infertile male. Fertility and sterility(1993)59: 393-396.

7. Arnold M. and Greg S. Transrectal prostate ultrasonography as a diagnostic and therapeutic aid for ejaculatory duct obstruction. J. Urol.1990; 144: 356-358.

8. Jack S. and Jacek L. Cyst of the ejaculatory duct/ urogenital sinus. J. Urol.1984; 132: 768-771.

9. Hendry W and Pryor J. Müllerian Duct (Prostatic Utricle) Cyst: Diagnosis and Treatment in Subfertile Males. British journal of urology(1992) 69:79-82.
10. Luxury T, Mcaninch W and Bunts C.Genital cysts of the male pelvis: case report of mullerian and ejaculatory duct cysts in the same patient.J. Urol.1973; 109: 440-443.

11. Xu B, Niu X, Wang A, Li P, Qin C, Li J, et al. Novel methods for the diagnosis and treatment of ejaculatory duct obstruction.British journal of urology(2010)108:263-266.

12. Eric TG, Steven AK, and Harry F. Seminal vesicle urinary reflux as a complication of transurethral resection of ejaculatory ducts. J. Urol.1995; 153: 1234-1235.

13. Benad Z, Goldwasser, John L, Weinerth, Culley C. Carson. Ejaculatory Duct Obstruction: The Case for Aggressive Diagnosis and Treatment. The Journal of Urology (1985) 134: 964-966.

14. Toshihiro Y, Tadaharu O, Atsuyuki Y, Masahiko U, Hiroyuki K, Yoshifumi K, et al. Cysts of the ejaculatory system: a report of two cases. Pediatr Surg Int (2005) 21: 939-942. 